\title{
Auf dem Weg zur zweckrationalen Relativität des Menschenwürdeschutzes*
}

In Fällen, in denen die Verfassungsmäßigkeit einer Grundgesetzänderung zur Debatte steht, besteht immer ein kompetenzielles Spannungsverhältnis zwischen dem parlamentarischen Gesetzgeber und dem Bundesverfassungsgericht. Die politische Entscheidung wird zu einem Auslegungsproblem, bei dem das BVerfG letztverbindlich über einen mit Zweidrittelmehrheit getragenen Beschluss der durch Wahlen direkt legitimierten Parlamentsabgeordneten entscheidet. ${ }^{1}$ Da Verfassungsauslegung jedoch zu einem gewissen Grade auch vom politischen Vorverständnis des Interpreten abhängig ist, birgt diese Konstellation demokratietheoretische Probleme. ${ }^{2}$ So ist zu erklären, dass das BVerfG bisher immer davor zurückgeschreckt ist, eine Verfassungsänderung mit dem Verdikt der Rechtswidrigkeit zu versehen. Diese Tendenz hat auch in dem Urteil des BVerfG zum sog. großen Lauschangriff ${ }^{3}$ ihre Bestätigung gefunden.

Gegenstand der Entscheidung war im wesentlichen eine Verfassungsänderung aus dem Jahre 1998. Zur Bekämpfung der organisierten Kriminalität hatte der Bundestag am 26. März 1998 mit den Stimmen der Fraktionen von CDU/CSU, SPD und FDP eine Änderung des Art. 13 GG beschlossen. Der neu eingefügte Art. 13 III GG sah eine Befugnis zur akustischen Überwachung von Wohnraum vor. Voraussetzung war der Verdacht einer besonders schweren Straftat, die Anordnung durch einen mit drei Richtern besetzten Spruchkörper sowie die Verhältnismäßigkeit, insbesondere die Erforderlichkeit der Maßnahme. Eine ähnliche Befugnis enthielt Art. 13 IV GG für die Gefahrenabwehr. Auf Basis dieser Grundgesetzänderung wurden in der StPO mehrere Ausführungsvorschriften zur Ermöglichung einer akustischen Wohnraumüberwachung erlassen. Der vorliegende Beitrag wird sich jedoch in erster Linie mit der verfassungsrechtlich interessanteren Frage der Rechtmäßigkeit der Änderung des Art. 13 GG auseinandersetzen. ${ }^{4}$ Hier berührt das Urteil im wesentlichen zwei zusammenhängende Problemkreise: zum einen den Maßstab bei der Änderung von Verfassungsrecht, zum anderen die Konkretisierung der Menschenwürdegarantie.

Im folgenden soll zunächst der vom Verfassungsgericht verwendete Prüfungsmaßstab erörtert werden (I.), ehe die Entscheidung des Verfassungsgerichts in materieller Hinsicht kurz skizziert (II.) und anschließend einer kritischen Würdigung unterzogen wird (III.).

\section{Prüfungsmaßstab}

Im Rahmen der Begründetheit war es erste Aufgabe des Gerichts, den Prüfungsmaßstab herauszuarbeiten. Eine Verfassungsänderung wie die Einfügung der Abs. 3-6 des Art. 13 GG kann nur dann Gegenstand einer rechtlichen Überprüfung sein, wenn innerhalb der Verfassung Normenhierarchien bestehen, also

* Zugleich Besprechung der Entscheidung des BVerfG zum großen Lauschangriff, Urt. v. 3. 3. 2004 -1 BvR 2378/98 -, NJW 2004, 999. Herzlicher Dank gilt Silja Vöneky und Thomas Diehn für Anregung und Kritik.

1 B.-O. Bryde, in: v. Münch/Kunig, Grundgesetz-Kommentar, Band 3, 4./5. Aufl. 2003, Art. 79, Rn. 25.

2 Eine Diskussion der möglichen Legitimitätsbasis des BVerfG findet sich bei U. Haltern, Integration als Mythos, JöR 45 (1997), $31 \mathrm{ff}$.

3 BVerfG, Urt. v. 3. 3. 2004 - 1 BvR 2378/98 -, NJW 2004, 999.

$4 \mathrm{Zu}$ den Ausführungen des BVerfG über die Veriassungsmäßigkeit der Ausführungsvorschriften in der StPO s. C. Gusy, Lauschangriff und Grundgesetz, JuS 2004, 457 (459 ff.). 
Rechtssätze existieren, an denen das gesamte übrige Verfassungsrecht zu messen ist. Eine solche Abstufung wird durch die Ewigkeitsgarantie des Art. 79 III GG vorgenommen, indem die Vorschrift die in Art. 1 und 20 GG niedergelegten Grundsätze für unabänderlich erklärt. ${ }^{5}$ Wäre nämlich eine Verfassungsänderung möglich, die mit den durch Art. 79 III GG geschützten Grundsätzen in Konflikt geriete, obwohl sie den Wortlaut von Art. 1 und 20 GG nicht verändert, könnten diese in ihrem materiellen Gehalt eingeschränkt werden, was die Ewigkeitsgarantie gerade zu vermeiden beabsichtigt.

Dagegen enthält die Wesensgehaltsgarantie des Art. 19 II GG keine Schranke für eine Grundrechtsänderung, ${ }^{6}$ da die Grundrechte in ihrem Bestand gerade nicht geschützt sind. Zwar wird argumentiert, dass alle Grundrechte in ihrem Kern einen Menschenwürdegehalt enthielten, der sich mit ihrem Wesensgehalt decke. ${ }^{7}$ Dann wäre Art. 19 II GG jedoch jeden eigenständigen Gehalts beraubt und hätte nur noch deklaratorischen Charakter. ${ }^{8}$ Außerdem kann die Verfassung durchaus Grundrechte gewähren, die von der Menschenwürde nicht geboten sind, aber dennoch einen Wesensgehalt besitzen. ${ }^{9}$ Daher ist für jedes Grundrecht gesondert zu prüfen, ob und inwieweit dessen Bestand unabdingbare Voraussetzung für die Gewährleistung der Menschenwürde ist. Pauschale Aussagen sind hier jedenfalls nicht möglich.

\section{Die Menschenwürdegarantie als zentraler Prüfungsmaßstab}

\section{a) Inbaltliche Konkretisierung der Menschenwürde}

Als zentralen Prüfungsmaßstab für den neuen Art. 13 III GG hat das BVerfG die Menschenwürdegarantie des Art. 1 I GG herangezogen. Über deren genauen Inhalt besteht bis heute kein Konsens. Im wesentlichen gibt es drei Ansätze einer positiven Begriffsbestimmung. ${ }^{10}$ So wird die Würde insbesondere in der christlichen Naturrechtslehre und der antiken Philosophie als Eigenwert des Menschen aufgefasst, durch den dieser sich von allen anderen Lebewesen unterscheide. ${ }^{11}$ Ein auf Niklas Lubmann zurückgehender Ansatz versucht dagegen eine eher relative Bestimmung der Menschenwürde. Der Mensch könne sich seine Würde allein durch Leistung erarbeiten, diese habe ihren Ursprung daher in seinem selbstbe-

5 Allgemeine Meinung: B. Pieroth, in: Jarass/Pieroth, Grundgesetz, 7. Aufl. 2004, Art. 79, Rn. 7; H. Dreier, in: ders. (Hrsg.), Grundgesetz, Band II, 1998, Art. 79 III, Rn. 11; J. Lücke, in: Sachs (Hrsg.), Grundgesetz, 2. Aufl. 1999, Art. 79, Rn. 22; K.E. Hain, in: v. Mangoldt/Klein/Starck, Das Bonner Grundgesetz, Band 3, 4. Aufl. 2001, Art. 79, Rn. 39.

6 H. Dreier, in: ders. (Hrsg.), Grundgesetz, Band I, 2. Aufl. 2004, Art. 1 I, Rn. 163; Bryde (Fn. 1), Rn. 36; M. Brenner, Möglichkeiten und Grenzen grundrechtsbezogener Verfassungsänderungen, dargestellt anhand der Neuregelung des Asylrechts, Der Staat 32 (1993), 493 (506 f.); J. Isensee, in: Isensee/Kirchhof (Hrsg.), HdbStR V, 2. Aufl. 2000, \$115, Rn. 71; K. Stern, Die Bedeutung der Unantastbarkeitsgarantie des Art. 79 III GG für die Grundrechte, JuS 1985, 329 (337).

7 G. Dürig, Zur Bedeutung und Tragweite des Art. 79 III des Grundgesetzes, in: Spanner u. a. (Hrsg.), FS Theodor Maunz, 1971, 41 (45 ff.); B. Raum/F. Palm, Zur verfassungsrechtlichen Problematik des »Großen Lauschangriffs«, JZ 1994, 447 (453).

8 B. Pieroth/B. Schlink, Grundrechte, 19. Aufl. 2003, Rn. 306.

9 Bryde (Fn. 1), Rn. 36; Dreier (Fn. 6), Rn. 164; Brenner (Fn. 6), 506 f.

$10 \mathrm{Zu}$ Ansätzen einer positiven Bestimmung der Menschenwürde s. die Überblicke bei Dreier (Fn. 6), Rn. 54 ff.; P. Häberle, in: Isensee/Kirchhof (Hrsg.), HdbStR I, 2. Aufl. 1995, $\$ 20$, Rn. 37 ff. Eine noch umfassendere Zusammenstellung und Kritik findet sich bei T. Geddert-Steinacher, Menschenwürde als Verfassungsbegriff, 1990, S. 112 ff.

11 S. dazu C. Starck, Menschenwürde als Verfassungsgarantie im modernen Staat, JZ 1981, 457 (459f.); J. Reiter, Menschenwürde als Małsstab, Aus Politik und Żeitgeschichte, B 23-24/2004, 6. 
stimmten Verhalten. ${ }^{12}$ Nach der Kommunikationstheorie schließlich konstituiert sich Würde durch soziale Anerkennung. ${ }^{13}$

Überwiegend wird die Menschenwürde heute jedoch nicht positiv durch eine Definition des zu schützenden Rechtsgutes, sondern vielmehr negativ, ausgehend von der verbotenen Eingriffshandlung bestimmt. ${ }^{14}$ Dies kommt auch in der vom BVerfG des öfteren bemühten, auf Kant ${ }^{15}$ zurückgehenden Objektformel zum Ausdruck, ${ }^{16}$ nach der eine Würdeverletzung dann vorliegt, wenn der Mensch nicht mehr auch »Zweck an sich selbst«, sondern nur noch bloßes Objekt staatlichen Handelns ist. ${ }^{17}$ Allerdings ist auch die Objektformel aufgrund ihrer Unbestimmtheit nur bedingt subsumtionstauglich. ${ }^{18}$ So wird eine Konkretisierung überwiegend durch die Bildung von Fallgruppen vorgenommen. Weitgehend anerkannt ist ein gewisser Kernbestand, etwa das Verbot von staatlicher Folter, ${ }^{19}$ von Akten gezielter Erniedrigung oder ethnischrassischer Diskriminierung und der Anspruch auf Gewährleistung des Existenzminimums. ${ }^{20}$

Jedoch bedarf auch die Herausbildung einer Kasuistik normativer Maßstäbe, was vor allem bei neu auftretenden Problemkonstellationen deutlich wird. Eine ausschließlich negative Bestimmung des Würdebegriffs stößt hier an ihre Grenzen. Wer bestimmte Handlungen als würdeverletzend qualifizieren will, muss - um sich nicht dem Vorwurf der Beliebigkeit auszusetzen - auch eine positive Vorstellung dessen haben, was menschliche Würde ausmacht. So ist auch Kants Objektformel letztlich nichts anderes als eine Negativformulierung des positiven Gebots, die Subjektqualität des Menschen zu achten, ${ }^{21}$ die wiederum durch die menschliche Autonomie konstituiert wird. ${ }^{22}$ Daher ist die Bestimmung des Würdebegriffs letztendlich nur unter Rekurs auf seine philosophischen oder gar metaphysischen Grundlagen möglich. ${ }^{23}$ Die negative Begriffsausprägung kann insoweit allein als Konkretisierung positiver Definitionsversuche verstanden werden.

\section{b) Unantastbarkeit der Menschenwürde}

Ein zweiter, aktueller Diskussionspunkt bezieht sich auf die Frage, ob die Menschenwürde unter bestimmten Voraussetzungen der Abwägung mit konkurrierenden Rechtsgütern zugänglich ist. Der überwiegende Teil der Literatur sieht eine

12 N. Lubmann, Grundrechte als Institution, 1965, S. 68 ff.

13 H. Hofmann, Die versprochene Menschenwürde, AöR 118 (1993), 353 (364). S. auch BVerfGE 27, 1 (6); 87, 209 (228).

14 B. Zypries, From Procreation to Generation? Constitutional and Legal-political Issues in Bioethics, in: Vöneky/Wolfrum (Hrsg.), Human Dignity and Human Cloning, 2004, 107; W. Höfling, in: Sachs (Fn. 5), Art. 1, Rn. 12; W. Graf Vitzthum, Die Menschenwürde als Verfassungsbegriff, JZ 1985, 201 (202). Zu ähnlichen Tendenzen bei der Herausbildung völkerrechtlichen Gewohnheitsrechts s. R. Wolfrum/S. Vöneky, Who is protected by Human Rights Conventions? Protection of the Embryo vs. Scientific Freedom and Public Health, in: Vöneky/Wolfrum, ebd., $133 \mathrm{ff}$.

15 I. Kant, Grundlegung zur Metaphysik der Sitten, S. 59 ff. (hrsg. von W. Weischedel, Werkausgabe, Band VII, 1974).

16 G. Luf, Menschenwürde als Rechtsbegriff, in: Zacyzk/Köhler/Kahlo (Hrsg.), Festschrift E.A. Wolff, 1998, 307 (319f.).

17 Zur Objektformel BVerfGE 9, 167 (171); 27, 1 (6); 72, 105 (116); 87, 209 (228). Grundlegend G. Dürig, Der Grundrechtssatz von der Menschenwürde, AöR 81 (1956), 117 (127).

18 Pieroth/Schlink (Fn. 8), Rn. 360; Höfling (Fn. 14), Rn. 14.

19 S. zu diesem jedoch noch unten, II $1 \mathrm{c}$.

20 Pieroth/Schlink (Fn. 8), Rn. 361; Hofmann (Fn. 13), 363.

21 C. Enders, Die Menschenwürde in der Verfassungsordnung. Zur Dogmatik des Art. 1 GG, 1997, S. 20.

22 Dazu Luf (Fn. 16), 309 ff.; F. Ekhardt, Würde und Existenzminimum - nur eingeschränkt für Asylbewerber?, ZAR 2004, 142 (143).

$23 \operatorname{Luf}$ (Fn. 16), 321; Siarck (Fn. 11), 463; E.-W. Bökenforde, Die Würde des Menschen war unantastbar, F.A.Z. v. 3. September 2003 , S. 33 . 
solche als generell unzulässig an. ${ }^{24}$ Schon der Wortlaut, der die Menschenwürde als unantastbar bezeichnet, spricht dafür, dass die Menschenwürde einer Abwägung mit anderen Rechtsgütern nicht zugänglich ist. ${ }^{25}$ Des weiteren enthält Art. 1 GG keine ausdrückliche Eingriffsermächtigung. Während andere vorbehaltlos gewährleistete Grundrechte ihre Schranke in konkurrierendem Verfassungsrecht finden, ${ }^{26}$ kann Art. 1 I GG wegen seines Schutzes durch Art. 79 III GG nicht durch andere Verfassungsnormen beschränkt werden. ${ }^{27}$ Diskutiert werden kann daher allenfalls, ob eine Einschränkung, die ihrerseits dem Schutz der Menschenwürde dient, gerechtfertigt sein kann. ${ }^{28}$

\section{c) Abrögungsthese}

Es gibt in der jüngeren Literatur allerdings Tendenzen, die die Unantastbarkeitsthese in Frage stellen. ${ }^{29}$ So schlägt Matthias Herdegen in seiner Kommentierung zu Art. 1 GG vor, zwischen einem unantastbaren Kernbereich und einem abwägungsoffenen Randbereich des Menschenwürdeschutzes zu unterscheiden. ${ }^{30}$ In den Kernbereich fielen solche Maßnahmen, bei denen entweder die modale Art der Behandlung (Beispiel: Genozid) oder der verfolgte Zweck (Beispiel: Diskriminierung aufgrund der Rasse) allein schon eine Würdeverletzung begründeten. Jedoch sei Gegenstand des Würdeanspruchs auch ein Schutz vor vollkommen unangemessenen staatlichen Eingriffen. Daher müsse neben dem Begriffskern des weiteren ein Begriffshof anerkannt werden, bei dem sich die Würdeverletzung gerade aus der Unverhältnismäßigkeit des gebrauchten Mittels zum verfolgten Zweck ergebe. ${ }^{31}$

Indem er die Abwägung somit schon zum Bestandteil der Begriffsbestimmung der Menschenwürde macht, immunisiert er seine These dogmatisch gegen den Einwand, dass für eine Eingriffsrechtfertigung eine ausdrückliche Ermächtigung fehle. Die Abwägung findet nicht auf Rechtfertigungs-, sondern bereits auf Schutzbereichsebene statt. Diese Konzeption besticht auf den ersten Blick durch zwei Merkmale: zum einen durch die scheinbar klare Unterscheidung zwischen Begriffshof und Begriffskern, zum anderen durch den Versuch einer dogmatischen Begründung dessen, was bereits übliche Rechtspraxis ist, ${ }^{32}$ einer Flexibilisierung des Würdeschutzes gegenüber dem starren Unantastbarkeitsdogma. ${ }^{33}$

Doch bereits der Fortgang der Kommentierung zeigt, dass eine Unterscheidung zwischen Begriffskern und Begriffshof nicht trennscharf möglich ist: So dürfe die Unzulässigkeit von Folter nicht rein modal beurteilt werden, sondern müsse immer im Zusammenhang mit dem verfolgten Zweck beurteilt werden. ${ }^{34}$ Die Diskussion um den Kernbereich der Menschenwürde wird somit auf die Frage vor-

C. Starck, in: v. Mangoldt/Klein/Starck, Das Bonner Grundgesetz, Band I, 4. Aufl. 1999, Art. 1, Rn. 30; Pieroth/Schlink (Fn. 8), Rn. 365; P Kunig, in: v. Münch/Kunig, Grundgesetz-Kommentar, Band 1, 4./5. Aufl. 2000, Art. 1, Rn. 4; W. Höfling, Die Unantastbarkeit der Menschenwürde - Annäherungen an einen schwierigen Verfassungsrechtssatz, JuS 1995, 857 (859).

25 Starck, ebd.; H.D. Jarass, in: Jarass/Pieroth (Fn. 5), Art. 1, Rn. 12.

26 Dazu K. Hesse, Grundzüge des Verfassungsrechts der Bundesrepublik Deutschland, 20. Aufl. 1995, Rn. 312.

27 F. Wittreck, Menschenwürde und Folterverbot, DÖV 2003, 873 (877); Kunig (Fn. 24), Rn. 4.

28 Vgl. zu dieser Frage Wittreck, ebd., 879 ff. einerseits und Pieroth/Schlink (Fn. 8), Rn. 365 andererseits.

29 Die im folgenden vorgestellte Konzeption Herdegens ist zwar der wohl prominenteste, nicht jedoch der einzige Versuch, Abwägungsgesichtspunkte in den Würdeschutz einzubeziehen. S. etwa auch Hofmann (Fn. 13), $365 \mathrm{ff}$. M. Herdegen, in: Maunz/Dürig, Grundgesetz (Lfg. 42, Stand: Februar 2003), Art. 1 Abs. 1, Rn. 43 ff. Ebd., Rn. 44.

32 S. dazu noch ausführlicher unten, III.

33 Diesen Vorteil hebt mit Blick auf das vorliegend besprochene Urteil auch Gusy (Fn. 4), $461 \mathrm{f}$. hervor.

34 Herdegen (Fn. 30), Rn.45. 
verlagert, ob eine bestimmte Handlung allein ihrer Modalität bzw. Finalität wegen schon eine Würdeverletzung darstellt. Das Beispiel verdeutlicht zudem, dass die Zweiteilung ein Instrument ist, um den Rand- zu Lasten des Kernbereichs immer weiter auszudehnen und so den Menschenwürdeschutz aufzuweichen. ${ }^{35}$ Eine Unterscheidung zwischen Begriffshof und Begriffskern ist daher kein geeigneter Ansatzpunkt, innerhalb des Schutzniveaus zu differenzieren.

Dogmatisch spricht jedoch noch ein weiteres Argument gegen die These Herdegens. Diese beruht auf der Prämisse, dass die vollkommene Unangemessenheit einer staatlichen Maßnahme für sich bereits Würdeverletzung begründen könne. Damit wird die Garantie des Art. 1 I GG in ihrer Struktur de facto derjenigen von Freiheitsgrundrechten mit einfachem Gesetzesvorbehalt angeglichen, ${ }^{36}$ wobei sich die tradierte dreistufige Prüfung auf der Ebene Schutzbereichsbestimmung abspielt. Das widerspricht jedoch der Intention des Verfassunggebers, der durch die Normierung der Menschenwürde ein gewisses ethisches Minimum dem gesetzgeberischen Zweckstreben bewusst entziehen wollte. ${ }^{37}$ Stützen lässt sich dies auch auf die Ausführungen Kants, für den sich die Menschenwürde von anderen Rechtsgütern gerade dadurch abhebt, das sie keinen Preis hat, also unbezahlbar und damit auch unaufwägbar ist. ${ }^{38}$ Dies ist denn auch die entscheidende Erkenntnis der kantischen Objektformel. Der Mensch wird nicht $d u r c h$ das mit staatlichem Handeln verfolgte Ziel zum Objekt; vielmehr besagt die Objektformel, dass alles Handeln, egal welchen Zweck es verfolgt, immer den menschlichen Eigenwert berücksichtigen müsse. ${ }^{39}$

\section{Die Lösung des Bundesverfassungsgerichts}

Das Bundesverfassungsgericht unterzieht die Verfassungsänderung in seiner Entscheidung zum Lauschangriff einer zweistufigen Prüfung. Erster Prüfungsmaßstab ist die Menschenwürdegarantie des Art. 1 I GG. Das Gericht stellt ausdrücklich fest, dass die Menschenwürde absolut geschützt und daher einer Abwägung mit konkurrierenden Rechtsgütern nicht fähig sei. ${ }^{40}$ Auf einer zweiten Stufe wird der Verhältnismäßigkeitsgrundsatz als weitere Schranke für diejenigen Maßnahmen herangezogen, die die Menschenwürdegarantie nicht verletzen. ${ }^{41}$ Diese Lösung des Bundesverfassungsgerichts unterscheidet sich von der soeben dargestellten Abwägungsthese dadurch, dass die Menschenwürde nicht in einen Kern- und einen Randbereich eingeteilt wird. ${ }^{42}$ Der Verhältnismäßigkeitsgrundsatz wird vielmehr nicht aus der Menschenwürde hergeleitet, sondern kommt erst dann zum Tragen, wenn der Schutzbereich des Art. 1 I GG bereits verlassen ist.

36 Der von Herdegen anerkannte Kernbereich entspräche dem durch Art. 19 II GG geschützten Wesensgehalt des Freiheitsrechts.

37 R. Spaemann, Begriff der Menschenwürde, in: Böckenförde/Spaemann (Hrsg.), Menschenrechte und Menschenwürde, 1987, 295 (307); Geddert-Steinacher (Fn. 10), S. 48.

38 Kant, GMdS (Fn. 15), S. 68. Dazu W. Graf Vitzthum, Back to Kant! An Interjection in the Debate on Cloning and Human Dignity, in: Vöneky/Wolfrum, Human Dignity (Fn. 14), 87 (96 ff.).

39 Spaemann (Fn. 37), 308.

40 BVerfG (Fn. 3), NJW 2004, 999 (1002).

41 Ebd.

42 S. zur Konkretisierung dieses Maßsstabes jedoch noch unten, îl 2. 
Eine Begründung der zweiten Schranke lässt das Gericht jedoch vermissen. In einer früheren Entscheidung, dem Abhörurteil, ${ }^{43}$ hatte das BVerfG eine Herleitung des Verhältnismäßigkeitsgrundsatzes als Maßstab für Verfassungsänderungen aus dem Rechtsstaatsprinzip angedeutet. ${ }^{44}$ Zwar ist in Art. 20 GG nicht ausdrücklich vom Rechtsstaatsprinzip die Rede. Normiert sind vielmehr einzelne Elemente. Allerdings lässt sich ein umfassender Schutz des Rechtsstaatsprinzips durch Art. 20 GG aus Art. 28 I 1 GG herleiten. Denn es ist kaum anzunehmen, dass die identitätsverbürgende Grundnorm für den Gesamtstaat in ihrem Gewährleistungsgehalt hinter der Homogenitätsklausel des Art. 28 GG zurückbleiben sollte. ${ }^{45}$

Bei der Frage des verfassungswidrigen Verfassungsrechts zeigt sich die rechtliche Relevanz der Diskussion, ob der Verhältnismäßigkeitsgrundsatz aus den Grundrechten oder dem Rechtsstaatsprinzip des Art. 20 GG herzuleiten ist. Nimmt man nämlich letzteres an, ist wegen Art. 79 III GG jede durch Verfassungsänderung in das Grundgesetz eingefügte Grundrechtsschranke am Prinzip der Verhältnismäßigkeit zu messen. ${ }^{46}$

Gegen eine Verankerung des Verhältnismäßigkeitsgrundsatzes im Rechtsstaatsprinzip könnte sprechen, dass es sich bei diesem um eine reine Verfahrensgarantie handelt, die ein ihr vorausgehendes Abwehrrecht zwingend erfordert. Am Maßstab der Verhältnismäßigkeit kann mithin nur ein Eingriff in ein bereits bestehendes Recht gemessen werden. Allerdings ist das Rechtsstaatsprinzip historisch gerade zum Schutz individueller Freiheit entwickelt worden. ${ }^{47}$ Dem gleichen Zweck dient der Verhältnismäßigkeitsgrundsatz. Selbst eine verfassungsändernde Mehrheit ist nicht legitimiert, über das zur Verfolgung ihrer Ziele erforderliche Maß hinaus in die Interessen der überstimmten Minderheit einzugreifen. Insofern wird die Wahrung der Verhältnismäßigkeit ein Gebot des Rechtsstaates. ${ }^{48}$

Löst man das Verhältnismäßigkeitsprinzip jedoch von den einzelnen Grundrechtsgewährleistungen und stellt es in den Schutz abstrakter Freiheit anstelle konkreter grundrechtlicher Werte, kommt ihm im Rahmen des Rechtsstaatsprinzips eine rein prozedurale Bedeutung zu. Dies muss auch Auswirkungen auf den Prüfungsumfang haben. Die Kontrolle beschränkt sich allein auf den legitimen Zweck, die Geeignetheit und die Erforderlichkeit einer Maßnahme. Eine Angemessenheitsprüfung, die ja im Kern eine Güterabwägung beinhaltet, findet dagegen nicht statt. ${ }^{49}$

43 BVerfGE 30, 1 (20f.).

44 Allerdings erscheint zweifelhaft, ob das BVerfG in der besprochenen Entscheidung diesen Weg wählen wollte, da es einen etwaigen, aus dem Rechtsstaatsprinzip hergeleiteten Verhältnismäßigkeitsgrundsatz noch einmal einer besonderen Würdigung unterzieht, s. BVerfG (Fn. 3), NJW 2004, 999 (1005). So scheint das BVerfG dem Verhältnismäßigkeitsprinzip nachgerade überpositiven Charakter zuweisen zu wollen.

45 H. Dreier, in: ders. (Hrsg.), Grundgesetz, Band 3, 1998, Art. 79 III GG, Rn. 44; E. Schmidt-Aßmann, in: Isensee/Kirchhof (Fn. 10), \$24, Rn. 3.

46 Insofern ist das Argument, einer Verankerung der Verhältnismäßigkeit im Rechtsstaatsprinzip bedürfe es nicht, da diese bereits aus den Grundrechten folge, nicht zutreffend. In diesem Sinne jedoch F.E. Schnapp, in: v. Münch/Kunig, Grundgesetz-Kommentar, Band 24./5. Aufl. 2001, Art. 20, Rn. 32; P. Kunig, Das Rechtsstaatsprinzip, 1986, S. 357.

47 R. Herzog, in: Maunz/Dürig, Grundgesetz (Stand: Lfg. 18, September 1989), Art. 20 VII, Rn. 12; M. Sachs, in: ders. (Fn. 5), Art. 20, Rn. 146; D. Merten, Zur verfassungsrechtlichen Herleitung des Verhältnismäßigkeitsprinzips, in: Hengstschläger u. a. (Hrsg.), FS Herbert Schambeck, 1994, 349 (372).

48 I.E. ebenso F. Ossenbühl, Maßhalten mit dem Übermaßverbot, in: Badura/Scholz (Hrsg.), FS Peter Lerche, 1993, 151 (155); K. Stern, Zur Entstehung und Ableitung des Übermaßverbots, in: Badura/ Scholz, ebd., $173 \mathrm{f}$.

49 Generell kritisch zur Angemessenheitsprüfung bei der rechtlichen Kontrolle des Gesetzgebers $B$. Schlink, Der Grundsatz der Verhältnismäßigkeit, in: Badura/Dreier (Hirsg.), FS 50 Jahre BVerfG II, 445 (461 f.). 
Ermächtigt der einfache Gesetzgeber zu einem Grundrechtseingriff, legitimiert sich die Angemessenheitsprüfung aus der Tatsache, dass ein höherrangiges Verfassungsgut eingeschränkt werden soll, was nur aufgrund einer entsprechenden verfassungsrechtlichen Ermächtigung zulässig ist. Die Prüfung der Proportionalität stellt sicher, dass die Grenzen der Ermächtigung eingehalten werden. ${ }^{50}$ Wird dagegen eine verfassungsrechtliche Grundrechtsschranke eingefügt, fehlt es an einem höherrangigen Rechtsgut, zu dessen Schutz eine Angemessenheitsprüfung vorgenommen werden könnte. ${ }^{51}$ Gleichrangige Rechtsgüter können in diese nicht eingestellt werden, da der verfassungsändernde Gesetzgeber schließlich nicht gehindert ist, die diesen zugrunde liegenden Normen zu ändern. Nähme man dennoch eine Angemessenheitsprüfung vor, würde man in Form der in die Abwägungsentscheidung eingestellten Güter neben den Art. 1 und 20 GG weitere Schranken anerkennen, die jedoch notwendigerweise überpositiven Ursprungs wären.

Damit ist der vom Verfassungsgericht herausgearbeitete Prüfungsmaßstab grundsätzlich zu begrüßen. Festzuhalten bleibt allerdings, dass bei Verfassungsänderungen eine Güterabwägung nicht stattfindet. Greift sie in die Menschenwürdegarantie ein, ist sie schon von vornherein unzulässig. Verletzt sie dagegen weder Menschenwürde noch Geeignetheits- und Erforderlichkeitsgrundsatz, ist sie zulässig, ohne dass es einer weiteren Proportionalitätsprüfung bedürfte.

\section{Unverletzlichkeit der Wohnung und Menschenwürdegehalt}

\section{Schutz des Kernbereichs privater Lebensgestaltung}

Bei der Konkretisierung der erarbeiteten Maßstäbe stellt das Verfassungsgericht fest, dass Art. 13 III GG zwar nicht gegen geltendes Verfassungsrecht verstoße. ${ }^{52}$ Allerdings müsse die Norm dafür verfassungskonform ausgelegt werden. Eine Maßnahme zur akustischen Wohnraumüberwachung unterliege neben den in Art. 13 III GG aufgeführten Kriterien weiteren Voraussetzungen, die direkt aus der Verfassung hergeleitet werden. Denn auch das Grundrecht der Unverletzlichkeit der Wohnung (Art. 13 I GG) sei eine Konkretisierung der Menschenwürdegarantie. ${ }^{53}$ So gehöre es zum Kern privater Lebensgestaltung, innere Empfindungen und intime Gefühle ohne Angst vor staatlicher Überwachung zum Ausdruck bringen zu können. Dafür benötige der Mensch einen geeigneten räumlichen Freiraum, die Privatwohnung als letztes Refugium. Zwar seien die Privaträume nicht absolut geschützt. Allerdings genieße in diesen dasjenige Verhalten Schutz, das »sich als individuelle Entfaltung im Kernbereich privater Lebensgestaltung darstellt. «" ${ }^{54}$ Dazu gehörten insbesondere »Gefühlsäußerungen, Äußerungen unbewussten Erlebens sowie Ausdrucksformen der Sexualität.«55 S. 360; ähnlich Schmidt-Aßmann (Fn. 45), Rn. 87.

51 Die Menschenwürdegarantie muss außer Betracht bleiben, da eine Verhältnismäßigkeitsprüfung ja ohnehin erst erfolgt, wenn Art. 1 I GG nicht berührt wird.

52 BVerfG (Fn. 3), NJW 2004, 999 (1000).

53 Ebd., 1002.

54 Ebd.

55 Ebd.

Ebd. Generiert durch IP '172.22.53.54', am 26.04.2023, 09:44:02 
Um den Schutz dieses »Kernbereichs privater Lebensgestaltung « zu gewährleisten, entwickelt das Verfassungsgericht im folgenden einen doppelten Kontrollmaßstab. Zunächst bestimmt sich die Rechtmäßigkeit der Maßnahme ex ante durch eine Wahrscheinlichkeitsprognose. Dabei ist die Möglichkeit einer Berührung des absolut geschützten Kernbereichs der Privatsphäre gegen die Wahrscheinlichkeit der Erlangung von Informationen über die verfolgte Straftat abzuwägen. Zu diesem Zweck stellt das Verfassungsgericht einen Kriterienkatalog auf, der sich hauptsächlich an der Art der zu überwachenden Räumlichkeit und den sich darin aufhaltenden Personen orientiert. ${ }^{56}$ Bei Geschäftsräumen bestünden geringere Eingriffsvoraussetzungen als bei Privatwohnungen; Gespräche mit nahen Verwandten und engsten Vertrauten verdienten größeren Schutz als sonstige soziale Kontakte. Halte sich der Beschuldigte demnach ausschließlich mit Personen, zu denen ein besonderes Vertrauensverhältnis besteht, in seiner Privatwohnung auf, sei eine Abhörmaßnahme nur zulässig, wenn es konkrete Anhaltspunkte gebe, dass die zu erwartenden Gespräche ihrem Inhalt nach unmittelbaren Bezug zu Straftaten aufwiesen. ${ }^{57}$

Erfüllt die Anordnung akustischer Wohnraumüberwachung diese Kriterien, unterliegt sie jedoch noch einer weiteren, einer ex post-Kontrolle. Führt die Abhörmaßnahme nämlich unerwartet doch zur Erhebung absolut geschützter Informationen, so müsse diese unverzüglich abgebrochen werden. Zudem seien die erlangten Daten sofort zu löschen..$^{58}$

\section{Kritik}

\section{Verfassungskonforme Auslegung von Verfassungsnormen}

Ein möglicher Kritikpunkt betrifft zunächst das methodische Vorgehen des Verfassungsgerichts. So kritisieren die Richterinnen Jaeger und Hohmann-Dennhart in ihrem Sondervotum die Figur der verfassungskonformen Auslegung von Verfassungsrecht, derer sich das BVerfG bedient, um zusätzliche ungeschriebene Voraussetzungen in Art. 13 III GG hineinzulesen und die Norm somit vor dem Verdikt der Verfassungswidrigkeit zu bewahren. Durch die Aufrechterhaltung der Norm würde der Schein einer zulässigen Grundrechtseinschränkung geschaffen, die den durch Art. 79 III GG gewährleisteten Standards nicht genüge. ${ }^{9}$

Funktion des Vorrangs der durch die Ewigkeitsgarantie geschützten Grundsätze gegenüber dem übrigen Verfassungsrecht ist jedoch, deren Aushöhlung zu vermeiden. ${ }^{60}$ Diesem Ziel ist bereits genügt, wenn die dafür erforderlichen zusätzlichen Schranken staatlicher Maßnahmen direkt aus den entsprechenden Verfassungsgrundsätzen hergeleitet werden. Nichts anderes passiert schließlich bei der - methodisch weitgehend anerkannten ${ }^{61}$ - verfassungskonformen Auslegung einfachgesetzlichen Rechts. Anders als die in der Verfassung normierten Grundrechtsschranken sind einfachgesetzliche Normen direkte Grundlagen von Grund-

61 S. nur K. Larenz/C.-W. Canaris, Niethodenlehre der Rechtswissenschaft, 3. Aufl. 1995, S. 159 ff. m. w. N. 
rechtseingriffen der Exekutive. Die Vermeidung des Scheins einer zulässigen Grundrechtseinschränkung wäre hier also viel dringender als auf Ebene der Verfassung.

Diese Überlegung mag den Senat dazu bewogen haben, den $\$ 100 \mathrm{~d}$ III StPO, in dem die formellen Voraussetzungen für die Anordnung von Maßnahmen zur akustischen Wohnraumüberwachung normiert sind, für verfassungswidrig zu erklären, da er die zuvor im Zusammenhang mit Art. 13 III GG entwickelten zusätzlichen Voraussetzungen nicht explizit enthalte. ${ }^{62}$

\section{Zur zweckrationalen Relativität der Menschenwürde}

Inhaltlich vermag die Entscheidung des Verfassungsgerichts den selbst definierten Anspruch jedoch nicht zu erfüllen. Allen Beteuerungen der Unantastbarkeit der Menschenwürde zum Trotz handelt es sich bei den Ausführungen zur Verfassungsmäßigkeit von Art. 13 III GG im Kern um eine reine Güterabwägung. ${ }^{63}$ Auf der einen Seite wird ein durch Art. 1 I GG absolut geschützter Bereich menschlichen Verhaltens definiert. Dessen Schutz wird jedoch auf der anderen Seite durch die Zulassung einer Prognoseentscheidung aufgeweicht, bei der die Anforderungen an die Anordnung der Überwachungsmaßnahme anhand der Wahrscheinlichkeit bestimmt wird, dass die Menschenwürde des Betroffenen tangiert wird. Damit wird, ähnlich wie im Polizeirecht, eine Abwägung von Gefahren für konkurrierende Rechtsgüter, die menschliche Würde und die Strafrechtspflege, vorgenommen. Die Qualifikation als Würdeverletzung wird von der Beurteilung der konkreten Maßnahme auf eine Überprüfung der hypothetischen Abwägung bei Anordnung der Maßnahme vorverlagert. Eine Überwachung, die in den eigentlich absolut geschützten Kernbereich eingreift, soll die Menschenwürde dann nicht verletzen, wenn die ursprüngliche Prognose nur sorgfältig genug getroffen worden ist. Dass dem Verfassungsgericht dieses Ergebnis nicht ganz behagt, wird durch die Einführung der ex post-Kontrolle ${ }^{64}$ deutlich. Ungeachtet dessen jedoch, dass diese praktisch wohl kaum handhabbar ist, ${ }^{65}$ kann diese eine etwaige Würdeverletzung in ihrer zeitlichen Intensität allenfalls mildern, nicht aber beseitigen.

Das BVerfG bestätigt damit eine Tendenz, die sich auch schon in früheren Entscheidungen gezeigt hat. So hat es etwa in seiner zweiten Tagebuchentscheidung ausgeführt, dass der Verwertung von Tagebuchaufzeichnungen im Strafprozess der durch Art. 1 I GG absolut geschützte Kernbereich des Persönlichkeitsrechts nicht entgegenstehe, wenn die Aufzeichnungen in engem Zusammenhang mit einer schweren Straftat stünden. ${ }^{66}$ Noch problematischer ist die Abhörentscheidung, in der das BVerfG - nach dem Motto: Der Zweck heiligt die Mittel - die Würdeverletzung allein von der Finalität der Maßnahme abhängig gemacht hat. ${ }^{67}$ Der Menschenwürdeschutz wird so im besten Fall zu einer reinen zweckrationalen Güterabwägung degradiert. Er fungiert nicht mehr als äußerste Grenze gesetzgeberischer Wertentscheidungen, sondern als Kontrollinstrument, das den

65 So etwa die Kritik v. $R$. Leicht, Karlsruher Widerspruch: Wanzen in Wohnungen erlaubt - Abhören intimer Gespräche verboten, Die Zeit v. 4. 3. 2004, S. 4.

66 BVerfGE 80, 367 (376f.). Ähnlich wie hier auch die Kritik von Starck (Fn. 24), Rn. 56; C. Roxin, Strafverfahrensrecht, 25. Aufl. 1998, $\$ 24$, Rn. 42; J. Wolter, Repressive und präventive Verwertung tagebuchartiger Aufzeichnungen, StV 1990, 175 (177).

67 BVerfGE 30, 1 (27): »Jedenfalls verletzt es die Menschenwürde nicht, wenn der Ausschluss des Gerichtsschutzes nicht durch eine Mißachtung oder Geringschätzung der menschlichen Person, sondern durch die Notwendigkeit der Geheimhaitung von Maßnahmen zum Schutze der demokratischen Ordnung und des Bestandes des Staates motiviert wird. "Hervorhebung hier. 
Verfassungsrichtern erlaubt, ihre eigene Güterabwägung an die Stelle derjenigen des verfassungsändernden Gesetzgebers zu setzen. Diese Aushöhlung des Grundrechtsschutzes unter Preisgabe dogmatischer Grundsätze ist leider kein Einzelfall: Auch in anderen Bereichen wird die Grundrechtsprüfung qualifizierter Grundrechtsschranken zum Trotz auf eine bloße Verhältnismäßigkeitskontrolle reduziert. ${ }^{68}$ Bleibt man etwa im Bereich des Art. 13 GG, ist die Entwicklung eines ungeschriebenen Rechtfertigungsgrundes für ordnungsbehördliche Besichtigungen in Betriebs- und Geschäftsräumen ${ }^{69}$ ein augenfälliges Beispiel.

\section{Intimsphäre und Menschenwürde}

Verständlich mag das Zögern des Verfassungsgerichts sein, den Spielraum des verfassungsändernden Gesetzgebers nicht übermäßig zu beschränken. Dogmatisch wäre zur Erreichung dieses Ziels ein restriktiveres Verständnis der Menschenwürde allerdings der einzig gangbare Weg. Die Ausführungen des Gerichts zu der Frage, inwieweit der Schutz der räumlichen Privatsphäre durch Art. 1 I GG geschützt ist, sind keineswegs zwingend. Das Verfassungsgericht stellt fest, dass der Leistungskraft der Objektformel im vorliegenden Fall Grenzen gesetzt seien. ${ }^{70}$ Anstelle des Versuches einer Entwicklung normativer Standards steht jedoch im folgenden eine rein intuitive Begründung. Auffällig ist etwa, dass der französische Conseil Constitutionnel bei einem fast zeitgleich ergangenen Urteil bei der Prüfung der Verfassungsmäßigkeit akustischer Wohnraumüberwachungsmaßnahmen eine Verletzung der Menschenwürde nicht einmal in Erwägung zieht. ${ }^{71}$ Auch wenn es sich bei dieser Entscheidung um ein entgegengesetztes Extrem handelt, ist sie doch Indiz genug, dass ein größerer Begründungsaufwand erforderlich gewesen wäre.

Ausgangspunkt der Bestimmung der Menschenwürde müssen wie dargestellt ${ }^{72}$ die positiven Definitionsansätze sein. Diese schließen sich dabei nicht gegenseitig aus, sondern können durchaus kumulativ verwendet werden. ${ }^{73}$ Herrschend sind die Theorien, die Würde als einen bestimmten Eigenwert des Menschen begreifen. Diesen leiten sie aus der Einzigartigkeit des Menschen gegenüber anderen Lebewesen her. ${ }^{74}$ Bereits in der antiken Philosophie wurde dieser Eigenwert durch Vernunftbesitz und Freiheit des menschlichen Willens konstituiert.

Beides kann dem Menschen jedoch nicht genommen werden. Der Mensch verliert seine Würde auch nicht durch Folter oder Erniedrigung. Was ihm genommen werden kann, ist lediglich die Möglichkeit der Außendarstellung der Würde. ${ }^{75}$ Auf die Außendarstellung hat sich folglich auch der Würdeschutz zu beziehen, so dass diesem immer intersubjektiver Bezug zukommt. In diesem Aspekt bestehen somit Berührungspunkte mit der Kommunikationstheorie, die Würde über den sozialen Achtungsanspruch des Menschen definiert.

S. nur S. Kadelbach, Ethik des Völkerrechts unter Bedingungen der Globalisierung, ZaöRV 2004, 1 (7). Vgl. jedoch auch F. Hufen, Erosion der Menschenwürde?, JZ 2004, 313 (314), der in Bezug auf die Menschenwürde zum genau entgegengesetzten Schluss kommt.

BVerfG, Urt. v. 17. 2. 1998 - 1 BvF 1/91 -, DVBl. 1998, 393 (397).

BVerfG (Fn. 3), NJW 2004, 999 (1001).

Entscheidung v. 2. 3. 2004 ( $\mathrm{n}^{\circ}$ 2004-492), Journal Officiel 2004, 4637, Rn. 62 ff. Allerdings ist einschränkend anzumerken, dass die Menschenwürdegarantie in der französischen Verfassung nicht ausdrücklich verankert ist, sondern vom Conseil Constitutionnel aus der fortgeltenden Präambel der Verfassung von 1946 entwickelt worden ist, s. dazu P. Rädler, Die Unverfügbarkeit der Menschenwürde in Deutschland und Frankreich, DÖV 1997, 109 (110).

S. oben, I 1 a.

Dreier (Fn.6), Rn. 58.

Starck (Fn. 11), 460.

Spaemann (Fn. 37), 299. Ähnlich allerdings mit anderer Konkretisierung Lubmann (Fn. 12), S. 68. 
Daher ist zu begrüßen, dass das BVerfG nicht die Wohnung an sich, in Form eines »Recht[s] auf Einsamkeit «, ${ }^{76}$ als vom Würdeschutz umfasst angesehen hat. ${ }^{77}$ Menschliche Autonomie und deren Außendarstellung sind nicht durch das Bestehen einer fest definierten räumlichen Abschirmungssphäre bedingt. ${ }^{78}$ Problematisch ist vielmehr die Heimlichkeit der Maßnahme. ${ }^{79}$ Durch diese verlieren die überwachten Personen die Kontrolle über den Adressatenkreis, dem sie intime Informationen anvertrauen. Die Kenntnis intimer Details ist jedoch geeignet, das Bild von einer Person und damit deren soziale Achtung erheblich zu beeinflussen. ${ }^{80}$ Dabei ist nicht entscheidend, ob die erlangten Informationen an eine breitere Öffentlichkeit gelangen, da sich der Anspruch auf Achtung der Würde gegen jedermann richtet. Daher liegt bereits in der Kenntnisnahme durch staatliche Organe und nicht erst in der konkreten Verwendung der erlangten Informationen ein Verstoß gegen Art. 1 I GG. ${ }^{81}$

\section{Fazit}

Die Entscheidung des BVerfG ist im Ergebnis ein Kompromiss: Die Richter konnten sich weder dazu durchringen, dem verfassungsändernden Gesetzgeber absolute Schranken zu setzen, noch wollten sie die politisch fragwürdige Entscheidung zu Gunsten eines großen Lauschangriffs sanktionslos passieren lassen. Verlierer dieses Kompromisses ist die tradierte Grundrechtsdogmatik, mit der die Entscheidung nicht in Einklang zu bringen ist. Da das Urteil damit jedoch mit einer Reihe früherer Entscheidungen auf einer Linie liegt, scheint die Hoffnung auf eine Kehrtwende derzeit illusorisch. Vielmehr zeigt sich eindrucksvoll, wie der in früheren Entscheidungen oft leichtfertige Umgang mit dem Begriff der Menschenwürde zu einer Absenkung des Schutzniveaus führen kann. ${ }^{82}$ Insofern ist Günter Dürigs Warnung, die Menschenwürde nicht als »kleine Münze« zu begreifen, ${ }^{83}$ heute aktueller denn je.

76 So aber Raum/Palm (Fn. 7), 451 unter Berufung auf BVerfGE 27, 1 (6).

77 S. BVerfG (Fn. 3), NJW 2004, 999 (1002).

78 Sähe man dies anders, wären etwa auch Massenunterkünfte für Asylbewerber mit dem Würdeschutz nicht vereinbar. Eine Abstufung des Würdeschutzes nach der Nationalität des Subjekts, wie sie Hofmann (Fn. 13), 366 f. vorschlägt, vermag angesichts der Tatsache, dass der Schutz des Art. 1 I GG nicht auf Deutsche beschränkt ist, nicht zu überzeugen. Umfassender gegen eine Abstufung des Würdeschutzes für Ausländer Ekhardt (Fn. 22), $144 \mathrm{ff}$.

79 Vgl. dazu auch Raum/Palm (Fn. 7), 451.

80 Insofern wird die Intimsphäre konsequenterweise auch überwiegend als Bestandteil des Würdeschutzes aufgefasst; s. etwa Dürig (Fn. 17), 129; E. Hilgendorf, Folter im Rechtsstaat, JZ 2004, 331 (337); R. Zippelius, in: Dolzer/Vogel/Graßhof (Hrsg.), Bonner Kommentar zum Grundgesetz (Stand: Lfg. 73, Dezember 1995), Art. 1 Abs. 1 u. 2, Rn. 97; Herdegen (Fn. 30), Rn. 86.

81 Anders in Bezug auf die Verwendung von Tagebuchaufzeichnungen Starck (Fn. 24), Rn. 56.

82 Zu diesem Zusammenhang Höfling (Fn. 24), 859.

83 Dürig (Fn. 17), 124. Ebenso E.-W. Böckenförde, Menschenwürde als normatives Prinzip, JZ 2003, 809 (811). 